\section{El aborto inducido y el riesgo de cáncer de mama: ¿existe una relación?}

El cáncer de la mama es una enfermedad común en los países desarrollados, donde una o dos de cada 20 mujeres adquieren la enfermedad en el transcurso de la vida. Las mujeres en países en desarrollo tienen un riesgo más bajo, pero la incidencia muestra una tendencia ascendente. El riesgo de sufrir la enfermedad parece ser mayor en mujeres de condición socioeconómica alta y en las que han tenido la menarquia a edad temprana, un primer parto y una menopausia a una edad avanzada, y antecedentes familiares de cáncer de mama.

Se han efectuado estudios para determinar si existe una asociación entre el riesgo de cáncer de mama y el aborto inducido. La mayor parte de ellos han sido estudios epidemiológicos de cohortes históricas o de casos y testigos, estos últimos basados en entrevistas sujetas a la fuerte influencia de sesgos de recordación. Los casos (es decir, las mujeres con cáncer de mama) suelen ser más propensos a proporcionar información fidedigna sobre el número de abortos inducidos porque tienen un interés personal en aportar datos para fines de la investigación; en cambio, los testigos, que casi siempre son mujeres sanas, no proporcionan esa información tan desinhibidamente. Ello podría conducir a una falsa elevación del riesgo calculado entre el cáncer de mama y el aborto inducido, aunque hasta ahora los resultados de los estudios de casos y testigos han sido muy poco congruentes.

Los estudios con cohortes históricas suelen verse menos afectados por limitaciones metodológicas de este tipo, y es alentador que los dos más extensos efectuados hasta la fecha no hayan arrojado ninguna asociación entre el aborto practicado en el primer trimestre de la gestación y el riesgo posterior de cáncer de mama. (World Health Organization. Induced abortion does not increase the risk of breast cancer. WHO Fact Sheet No. 240, June 2000.)

\section{Los trastornos psiquiátricos en trabajadores itinerantes mexicanos}

Casi la mitad de toda la población que se dedica a labores agrícolas en los Estados Unidos de América está constituida por trabajadores itinerantes procedentes de otros países, y de esta mano de obra extranjera, prácticamente $90 \%$ proviene de México. Se trata de una población compuesta principalmente de hombres menores de 40 años, casi siempre padres de familia, que viven en condiciones muy precarias y devengan un ingreso medio de US\$ 2500 a \$5 000 anuales. Pese a su pobreza, estas personas raras veces acuden a los servicios de asistencia social.

Diversos estudios han revelado una mayor prevalencia de trastornos mentales, alcoholismo y abuso de drogas entre los trabajadores itinerantes de algunos países que en el común de la población local; otros han mostrado lo contrario, es decir, que los itinerantes tienen mayor estabilidad mental que la población local y que la población general de su país de origen. Tal ha sido el caso de los trabajadores portugueses en Suiza y de los trabajadores itinerantes en los países de Europa Occidental.

Es indudable que se trata de un tema de gran complejidad, dada la gran variedad de factores que ejercen su influencia en la salud mental. Por una parte, las malas condiciones en que viven los trabajadores itinerantes los colocan en mayor riesgo de sufrir trastornos mentales; por otra, los trabajadores que provienen de países donde la prevalencia de estos trastornos es baja en general podrían estar en mejor estado emocional que la población del país donde trabajan, gracias al efecto protector de ciertos factores sociales y culturales propios de la sociedad de origen.

En un condado del estado de California, un grupo de investigadores estudió la prevalencia de 12 trastornos psiquiátricos en 1001 campesinos itinerantes mexicanos de ambos sexos. La muestra fue seleccionada por conglomerados, y se aplicó una versión modificada de un cuestionario estándar, el Composite International Diagnostic Interview, para identificar los casos. Se exploraron los efectos de factores sociodemográficos y del proceso de adaptación a la nueva sociedad sobre la frecuencia de trastornos psiquiátricos en el transcurso de toda la vida.

Se detectaron las siguientes tasas de incidencia: hombres, 26,7\% (error estándar [EE] = 1,9); mujeres, $16,8 \%(\mathrm{EE}=1,7)$; indígenas: $26,0 \%(\mathrm{EE}=4,5)$; no indígenas, 20,1\% $(\mathrm{EE}=1,3)$. Los porcentajes de trastornos específicos detectados en toda la muestra fueron los siguientes: trastornos afectivos, 5,7\%; ansiedad, 12,5\%; abuso o dependencia de sustancias, $8,7 \%$; personalidad antisocial, $0,2 \%$. La prevalencia de cualquier trastorno psiquiátrico en el transcurso 
de la vida fue menor entre los trabajadores itinerantes mexicanos que en las poblaciones mexicanoestadounidense y estadounidense en general. El riesgo de padecer estos trastornos mostró un aumento en aquellos trabajadores que más se habían adaptado a las costumbres del nuevo país y que pasaban la mayor parte del tiempo en los Estados Unidos. (Alderete E, Vega WA, Kolody B, Aguilar-Gaxiola S. Lifetime prevalence of and risk factors for psychiatric disorders among Mexican migrant farmworkers in California. Am J Public Health 2000;90(4): 608-614.)

\section{Efecto del ejercicio en el riesgo de accidentes cerebrovasculares en mujeres}

Las pruebas son fehacientes: la actividad física reduce notablemente el riesgo de enfermedad coronaria. Pero ies también capaz de aminorar el riesgo de accidentes cerebrovasculares? Los pocos estudios epidemiológicos efectuados hasta la fecha han arrojado resultados contradictorios. Ninguno ha detectado una asociación muy clara entre la disminución del riesgo y la frecuencia y duración del ejercicio. El efecto del ejercicio físico en los distintos tipos de accidentes cerebrovasculares no ha sido debidamente investigado, y la mayoría de los estudios que se han llevado a cabo se han centrado en la población masculina. Según los resultados del estudio de Framingham, la actividad física intensa confiere protección contra los accidentes cerebrovasculares en general en hombres, pero no en mujeres.

Una cohorte de 72488 enfermeras estadounidenses entre las edades de 40 y 65 años quienes en 1986 no mostraban signos de enfermedad cardiovascular o cáncer fue observada a lo largo de 8 años con el fin de determinar la incidencia de distintos tipos de accidentes cerebrovasculares. El ejercicio físico se midió en quintiles y se basó en el número de horas semanales dedicadas a tareas con un gasto metabólico equivalente (metabolic equivalent tasks, MET). Hubo un total de 560087 años-persona de observación. Se documentaron 407 accidentes cerebrovasculares (258 de carácter isquémico, 67 hemorragias subaracnoideas, 42 hemorragias intracerebrales, y 40 de tipo indefinido). Cuando se efectuaron análisis multivariantes con ajustes por edad, índice de masa corporal, antecedentes de hipertensión y otras covariables, se detectó una fuerte asociación inversa entre la cantidad de actividad física y el riesgo de accidente cerebrovascular en general. Los riesgos relativos para cada quintil de ejercicio fueron 1,00 (grupo de referencia); 0,98; 0,82; 0,74; y 0,66 ( $P$ de la tendencia $=0,005)$. La gradiente inversa se observó principalmente en el caso de accidentes cerebrovasculares isquémicos. No se detectó ninguna asocia- ción significativa entre la actividad física y la hemorragia subaracnoidea o la hemorragia intracerebral. Aun después de hacer ajustes por las distintas variables, el ejercicio de caminar a una velocidad normal se asoció con un riesgo reducido de accidentes cerebrovasculares en general y de carácter isquémico en particular. El hecho de que las mismas asociaciones se hayan advertido, en mayor grado aun, con el ejercicio de caminar a paso largo o acelerado indica que el efecto protector guarda relación directa con la cantidad e intensidad del ejercicio. (Hu FB, Stampfer MJ, Colditz GA, Ascherio A, Rexrode KM, Willett WC, et al. Physical activity and risk of stroke in women. JAMA 2000;283(22): 2961-2967.)

\section{Se propaga la tuberculosis pese a las medidas de control recomendadas}

Los adelantos que se han logrado en el último decenio en el campo de la epidemiología molecular han ayudado a dilucidar aspectos previamente desconocidos de la dinámica de la transmisión de Mycobacterium tuberculosis. Pero pese a estos conocimientos y a la adopción de medidas de control, la tuberculosis se sigue propagando. Se han documentado numerosos brotes de la enfermedad en hospitales, casas de reposo, albergues para los desposeídos y otras instituciones donde la transmisión se produce por contacto con personas infectadas. Hasta la fecha no se ha publicado ningún informe que explique los motivos por los cuales las intervenciones de salud pública efectuadas hasta ahora no han dado los resultados esperados. Los datos obtenidos mediante este tipo de análisis podrían servir para orientar el diseño y la implantación de futuras intervenciones.

Desde 1996, un grupo de investigadores cerca de San Francisco, California, ha venido efectuando un estudio basado en un conglomerado de 73 pacientes infectados con la misma cepa de $M$. tuberculosis, de un total de 221 casos notificados en el mismo condado. Los investigadores sabían que en 1992 y 1993 varios pacientes de la zona habían estado infectados con esa misma cepa bacteriana, hecho confirmado mediante técnicas de epidemiología molecular, y procedieron a identificar los factores que habían facilitado su propagación.

De los 73 pacientes, 39 (53\%) habían adquirido la enfermedad porque nunca fueron identificados como contactos de los casos iniciales; 20 (27\%) se enfermaron porque hubo demora en llegar al diagnóstico del caso original; 13 (18\%) adquirieron la tuberculosis debido a dificultades con la evaluación o el tratamiento de los contactos; y 1 paciente $(1 \%)$ se enfermó debido a la tardanza en ser identificado como contacto. 
Estos resultados apuntan al hecho de que aun en comunidades donde se han implantado los elementos esenciales del control de la tuberculosis, seguirán produciéndose casos a no ser que el diagnóstico de los pacientes se haga más temprano y que la identificación de los contactos sea más exhaustiva. (Chin DP, Crane CM, Ya Diul M, Sun SJ, Agraz R, Taylor S, et al. Spread of Mycobacterium tubrculosis in a community implementing recommended elements of tuberculosis control. JAMA 2000;283(22):2968-2974.)

\section{Efectos de la fertilización in vitro sobre los resultados del embarazo}

El primer niño en ser producto de una fertilización in vitro nació en 1978. El éxito de esta técnica suele ser medido en términos de la tasa de embarazos, pero el resultado de mayor importancia es, en realidad, el estado de salud del neonato. Ahora que se ha logrado una mayor tasa de supervivencia en niños con bajo peso al nacer, es imprescindible poder entender el papel que actualmente desempeña la fertilización in vitro en la duración del embarazo y el peso y otras características del neonato. Los estudios en este sentido han sido muy escasos; se sabe muy poco acerca de la contribución de la fertilización in vitro a la incidencia poblacional de bajo peso al nacer $(<2500 \mathrm{~g})$, parto prematuro (<37 semanas de gestación), peso muy bajo al nacer (<1500 g) o embarazos múltiples.

Aunque teóricamente un embarazo logrado por fertilización in vitro no tiene por qué diferir de un embarazo natural después de la concepción, ha habido informes de un riesgo elevado de aborto espontáneo y de morbilidad y mortalidad neonatales en el caso de embarazos logrados artificialmente. Un grupo de investigadores se ha dedicado a la tarea de tratar de determinar en qué medida la fertilización in vitro ha afectado a la frecuencia poblacional de diversos trastornos neonatales y gestacionales en el período de 1994 a 1996 en Alberta, Canadá. Revisaron las estadísticas correspondientes a ese período y encontraron que la fertilización in vitro es responsable de $17,8 \%$ del aumento observado de bajo peso al nacer; $43,5 \%$ del aumento de peso muy bajo al nacer; $10,5 \%$ y $66,2 \%$ del aumento de neonatos con menos de 37 y de 30 semanas de edad gestacional, respectivamente; y $21,4 \%$ de todos los nacimientos gemelares y triples documentados en la provincia. Estas son contribuciones bastante notables y su importancia se ve resaltada por el hecho de que el peso y la edad gestacional al nacer son los dos factores que ejercen mayor influencia en las tasas de mortalidad y morbilidad de los recién nacidos y niños pequeños. (Tough SC, Greene CA, Svenson LW, Belik J. Effects of in vitro fertilization on low birth weight, preterm delivery, and multiple birth. J Pediatr 2000;136(5): 618-622.)

\section{La doble fortificación de la sal de mesa mata dos pájaros de un tiro}

Al cabo de cinco años de investigaciones y pruebas dirigidas por la Universidad de Toronto, Canadá, en Bangladesh, Ghana y Guatemala, se ha logrado elaborar una sal estable fortificada con hierro y con yodo que goza de la aceptación de los consumidores. Hoy en día el mundo se enfrenta a una tragedia prevenible: la desnutrición por carencia de micronutrientes. El hierro y el yodo se encuentran en cantidades insuficientes en la alimentación de más de mil millones de personas en países en desarrollo.

Ha habido varias iniciativas destinadas a fortificar los alimentos con micronutrientes que escasean en la dieta de poblaciones pobres y mal nutridas. En la mayor parte de los países, son pocos los alimentos básicos de consumo general - cereales, aceites, azúcar y sal-que se prestan para la fortificación. Hay territorios donde la población consume sal a pesar de que es el único alimento que no se produce localmente. De ahí que la sal haya sido el alimento elegido para la fortificación con yodo, la cual se ha logrado en prácticamente el mundo entero en cuestión de un decenio.

No es nueva la idea de agregarle a la sal de consumo hierro, además de yodo, en un esfuerzo por combatir a un mismo tiempo la anemia ferropénica y el bocio, pero una de las dificultades ha consistido en evitar la incompatibilidad química entre las dos sustancias al mezclarlas, que redundaría en la pérdida de nutrientes. Tampoco ha sido fácil lograr una sal de buena calidad que sea estable en distintos envases $\mathrm{y}$ diferentes condiciones de almacenamiento.

La sal fortificada con yodo y hierro es un producto de gran pureza elaborado con fumarato ferroso y yoduro de potasio recubiertos de dextrina. El encapsulamiento impide que se produzca interacción entre el hierro y el yodo y que estos elementos se humedezcan al entrar en contacto con la sal. La mezcla de ingredientes se lleva a cabo en un ambiente seco y a una velocidad específica. Posteriormente, la sal se coloca en envases a prueba de humedad, medida indispensable si se ha de mantener la estabilidad de los nutrientes. La sal elaborada con esta técnica ya ha pasado las cuatro pruebas esenciales: estabilidad, aceptabilidad, eficacia y factibilidad comercial. Se conserva más de un año en diferentes condiciones de humedad y almacenamiento sin que sufra ninguna alteración más que un ligero cambio de coloración que no parece molestar a los consumidores. Los resultados de un estudio 
clínico en Ghana han demostrado que esta sal es tan eficaz como un suplemento semanal de hierro en la prevención de anemia en la mujer; también previene y corrige la anemia en niños, así como la deficiencia de yodo en niños y en mujeres. Por último, la mayoría de los países cuentan con los medios para producir la mezcla de hierro y yodo encapsulados y distribuirla a sus respectivos fabricantes de sal. Una vez que se haya simplificado un poco la tecnología, proceso que ya se ha iniciado, la sal podrá producirse a escala comercial a un costo de alrededor de 2 a 3 centavos de dólar estadounidense por kilogramo. (The Micronutrient Initiative. Double fortification of salt. Comunicado accesado en el sitio http://www.micronutrient.org de la Internet el 9 de mayo de 2000.)

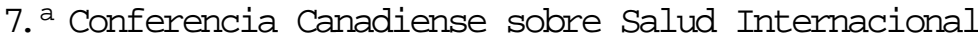
(7th Canadian Conference on International Health)

Fechas: 12 a 15 de noviembre de 2000

Lugar: Ottawa/Hull, Ontario, Canadá

Tema: La salud como puente a la paz en el mundo (Health as a bridge to world peace)

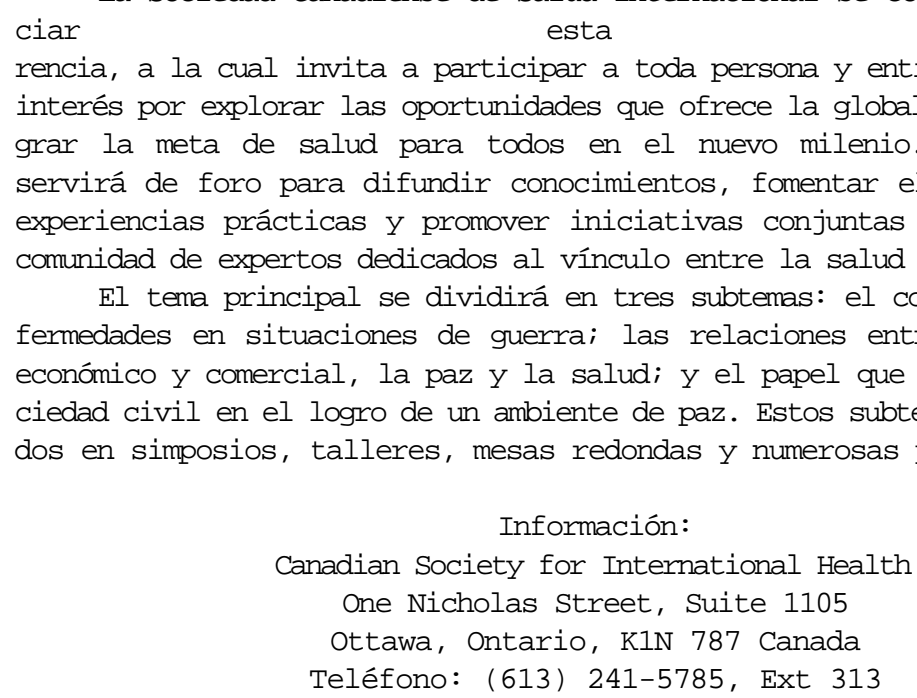

